Pacific Journal of Mathematics

FACTORIZATION OF RADONIFYING TRANSFORMATIONS 


\title{
FACTORIZATION OF RADONIFYING TRANSFORMATIONS
}

\section{Robert Fernholz}

\begin{abstract}
It is shown that a linear transformation which carries a cylinder measure on a separable Hilbert space to a Radon measure on a separable Banach space can be factored into a positive-definite Hilbert-Schmidt transformation followed by a measurable linear transformation. Applications to measurable norms are given.
\end{abstract}

1. Introduction. It is well known that Hilbert-Schmidt transformations carry certain well behaved cylinder measures into Radon measures on Hilbert spaces (see [8, 9]). The problem of characterizing transformations which carry cylinder measures on Hilbert spaces to Radon measures on Banach spaces seems to be more difficult (see [8]). In this paper it is shown that such a transformation can be factored into a positive-definite Hilbert-Schmidt transformation followed by a measurable linear transformation. In the last section this type of factorization is applied to abstract Wiener spaces.

2. Radon measures on embeddable spaces. Through. out this paper all topological vector spaces (TVS) will be assumed to be real and locally convex. A Radon measure is a regular Borel measure, and we shall assume that all Radon measures in this paper are positive with total measure 1 , i.e. they are probability measures. A topological space $X$ is a Lusin space if there is a complete separable metric space $Y$ and a continuous bijective mapping $Y \rightarrow X$. Any Borel measure on a Lusin space is a Radon measure ([9], p. 122). The Borel subsets for comparable Lusin topologies are identical ([9], p. 101).

DEFINITION 2.1. A TVS $E$ is embeddable if $E$ is a Lusin space and if there is a continuous linear injection $T: E \rightarrow H$ where $H$ is a separable Hilbert space.

Any such mapping $T$ will be called an embedding of $E$. We can assume that $T(E)$ is dense in $H$. Kuelbs [4] has shown that any separable Banach space is embeddable. In fact we have

LEMMA 2.2. A TVS is embeddable if and only if it is a Lusin space and there exists a countable bounded set $\left\{y_{\imath}\right\} \subset E^{\prime}$, the dual of $E$, which separates points on $E$. 
Proof. If $T: E \rightarrow H$ is an embedding, then $T^{*}: H \rightarrow E^{\prime}$, the adjoint of $T$, is bounded and for any orthonormal basis $\left\{x_{i}\right\}$ of $H,\left\{T^{*} x_{i}\right\} \subset E^{\prime}$ separates points on $E$.

Conversely if $\left\{y_{i}\right\} \subset E^{\prime}$ is a countable bounded subset which separates points and $H$ is any separable Hilbert space with orthonormal basis $\left\{x_{i}\right\}$, the transformation $T: E \rightarrow H$ defined by

$$
T x=\Sigma t_{i}\left\langle x, y_{i}\right\rangle x_{i}, \quad x \in E,
$$

where $t_{i}>0, \Sigma t_{i}^{2}<\infty$, is an embedding. $(\langle$,$\rangle is the canonical bilinear$ form on $E \times E^{\prime}$.)

Lemma 2.3. Let $E$ be embeddable with embedding $T: E \rightarrow H$. Then for any Borel subset $B \subset E, T(B)$ is Borel in $H$.

Proof. This is true of injective mappings on Lusin spaces ([9] p. 107, Lemma 14).

Definition 2.4. Let $H$ be a separable Hilbert space with Radon measure $\mu$. Let $A$ be a linear transformation defined on a dense linear subspace $\mathscr{D}_{\mathrm{A}} \subset H$ with image in a TVSE. $A$ is a $\mu$-measurable linear transformation if $\mu\left(\mathscr{D}_{A}\right)=1$ and for any Borel set $B \subset E, A^{-1}(B)$ is Borel.

We shall use the notation $A: H \rightarrow E$ even when $\mathscr{D}_{A} \neq H$. Any bounded linear transformation is $\mu$-measurable. $A \mu$-measurable linear transformation $A: H \rightarrow E$ induces a Borel measure $A(\mu)$ on $E$ defined by $A(\mu)(B)=\mu\left(A^{-1}(B)\right)$ for any Borel set $B \subset E$.

Lemma 2.5. Let $E$ be an embeddable space with embedding $T: E \rightarrow H$, and let $\mu$ be a Radon measure on $E$. Then $A=T^{-1}: H \rightarrow E$ is $T(\mu)$-measurable.

Proof. $T(\mu)$ is a Borel measure and therefore a Radon measure on H. $\quad T(\mu)\left(\mathscr{D}_{A}\right)=T(\mu)(T(E))=1$. For any Borel set $B \subset E, A^{-1}(B)=$ $T(B)$ is Borel in $H$ by Lemma 2.3 , so $A$ is $T(\mu)$-measurable.

A cylinder measure $\gamma$ on a Hilbert space $H$ is scalarly concentrated on the balls of $H$ if for all $\epsilon>0$ there is $N>0$ such that for any $y \in H$, $\|y\|=1$,

$$
\gamma\{x:|\langle x, y\rangle| \leqq N\}>1-\epsilon
$$

where $\langle$,$\rangle denotes the inner product on H$. We shall assume that all cylinder measures discussed in this paper have this property. 
Lemma 2.6. Let $H_{1}, H_{2}$ be Hilbert spaces, $S: H_{1} \rightarrow H_{2}$ be a Hilbert-Schmidt transformation, and $\gamma$ be a cylinder measure on $H_{1}$. Then $S(\gamma)$ can be extended to a Radon measure $\overline{S(\gamma)}$ on $H_{2}$.

Proof. See [9] p. 301, or [8].

THEOREM 2.7. Let $E$ be an embeddable TVS, $H$ be a separable Hilbert space, $S: H \rightarrow E$ be a bounded linear transformation, and $\gamma$ be a cylinder measure on $H$. Then $S(\gamma)$ can be extended to a Radon measure on $E$ if and only if $S$ can be factored

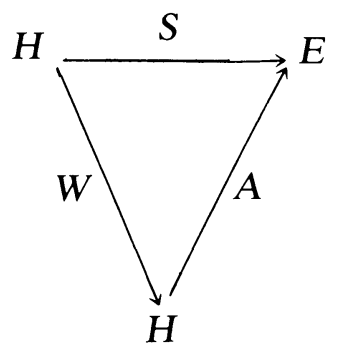

where $W$ is a positive-definite Hilbert-Schmidt transformation and $A$ is a closed $\overline{W(\gamma)}$-measurable linear transformation.

Proof. If $S$ can be so factored, then $A \overline{(W(\gamma))}$ will be a Radon measure on $E$. Since $\overline{A(W(\gamma))}$ and $S(\gamma)$ agree on cylinder sets, $S(\gamma)$ can be extended to $\overline{A(W(\gamma))}$.

Conversely, let $T: E \rightarrow H_{1}$ be an embedding. Since $H$ and $H_{1}$ are both separable, they are unitarily equivalent, so we can assume $T: E \rightarrow H$. If necessary $T$ can be followed by a bijective Hilbert-Schmidt transformation and still remain an embedding, so we can assume that $W=T S$ is Hilbert-Schmidt, and in fact that $W$ is positive-definite.

$\overline{W(\gamma)}=T \overline{(S(\gamma))}$ and $A=T^{-1}: H \rightarrow E$ is $\overline{T(S(\gamma))}$-measurable by Lemma 2.5. $T$ is continuous so $A=T^{-1}$ is closed.

REMARK. Theorem 2.7 cannot be improved to state that $S=A W$ where $W$ is Hilbert-Schmidt and $A$ is bounded (see [6] p. 107 and [8] Proposition (5.20.4)).

The following corollary resembles Theorem 1 of Versik [10], but without the hypothesis that $(E, \mu)$ be a "Lebesgue space".

COROllary 2.8. Let $E$ be an embeddable space with Radon mea. 
sure $\mu$. Then there exists a separable Hilbert space $H$ with Radon measure $\nu$ and a bijective $\nu$-measurable linear transformation $A: H \rightarrow E$ such that $\mu=A(\nu)$.

\section{Application to sequentially measu rable norms.}

Definition 3.1. Let $H$ be a separable Hilbert space and $\gamma$ be a cylinder measure on $H$. A continuous norm \|\| on $H$ is sequentially $\gamma$-measurable if for any increasing sequence of finite dimensional orthogonal projections $\left\{P_{n}\right\}$ on $H$ such that $P_{n} \rightarrow I_{H}$ and $\epsilon>0$, there is $N>0$ such that for $m, n>N, \gamma\left\{x:\left\|P_{n} x-P_{m} x\right\|>\epsilon\right\}<\epsilon$.

$I_{H}$ denotes the identity operator on $H$. To avoid confusion we shall denote the original (Hilbert) norm on $H$ by \|\|$_{H}$.

If $\gamma$ is the standard Gaussian cylinder measure on $H$, then a measurable norm (defined in [2,3]) is also sequentially $\gamma$-measurable ([2] Corollary 5.2).

THEOREM 3.2. (Gross). Let $\gamma$ be a cylinder measure on a separable Hilbert space $H$ and let \|\| be a sequentially $\gamma$-measurable norm on $H$. Let $B$ denote the Banach space generated by $H$ with norm \|\| and let $S: H \rightarrow B$ be the natural injection. Then $S(\gamma)$ can be extended to a Radon measure on $B$.

Proof. Since $H$ is separable, $B$ is separable and therefore embeddable by Lemma 2.2. Let $T: B \rightarrow H$ be an embedding such that $T S$ is a positive-definite Hilbert-Schmidt transformation. TS $(\gamma)$ can be extended to a Radon measure $\nu$ on $H$. We must show that the closed linear transformation $A=T^{-1}: H \rightarrow B$ is $\nu$-measurable which, in view of Lemma 2.3, amounts to showing that $\nu\left(\mathscr{D}_{A}\right)=1$.

Let $\left\{P_{n}\right\}$ be an increasing sequence of finite dimensional orthogonal projections on $H$ such that $P_{n} \rightarrow I_{H}$ and which commute with TS. Then for $\epsilon>0$

$$
\begin{aligned}
\gamma & \left\{x:\left\|P_{n} x-P_{m} x\right\|>\epsilon\right\} \\
& =\gamma\left\{x:\left\|S P_{n} x-S P_{m} x\right\|_{B}>\epsilon\right\} \\
& =\gamma\left\{x:\left\|A T S P_{n} x-A T S P_{m} x\right\|_{B}>\epsilon\right\} \\
& =\gamma\left\{x:\left\|A P_{n} T S x-A P_{m} T S x\right\|_{B}>\epsilon\right\} \\
& =\nu\left\{x:\left\|A P_{n} x-A P_{m} x\right\|_{B}>\epsilon\right\}
\end{aligned}
$$


Therefore, if $m$ and $n$ are large enough,

$$
\nu\left\{x:\left\|A P_{n} x-A P_{m} x\right\|_{B}>\epsilon\right\}<\epsilon
$$

so $A P_{n} x$ converges in $\nu$-measure. Then a subsequence, also denoted by $A P_{n} x$, converges $\nu$-almost everywhere. Since $A$ is closed and $P_{n} x \rightarrow x$ we must have $A P_{n} x \rightarrow A x \nu$-almost everywhere. Therefore $\nu\left(\mathscr{D}_{A}\right)=1$.

THEOREM 3.3. Let $\gamma$ be a cylinder measure on a separable Hilbert space $H$ and let \|\| be a sequentially $\gamma$-measurable norm on $H$. Let $B$ denote the Banach space generated by $H$ with norm \|\| , let $S: H \rightarrow B$ be

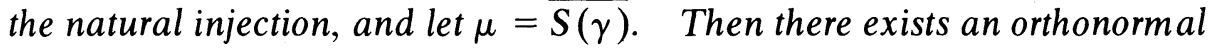
basis $\left\{e_{k}\right\}$ of $H$ with $e_{k}=S^{*} f_{k}, f_{k} \in B^{\prime}$, such that

$$
\sum_{k=1}^{n}\left\langle x, f_{k}\right\rangle S e_{k} \rightarrow x
$$

in $\mu$-measure on $B$.

Proof. As in the proof of Theorem 3.2 we can define an embedding $T: B \rightarrow H$ such that $W=T S$ is a positive-definite Hilbert-Schmidt transformation and $A=T^{-1}: H \rightarrow B$ is a closed $\overline{W(\gamma)}$-measurable linear transformation. Let $\left\{e_{k}\right\}, k \in \mathbf{N}$, be an orthonormal basis of $H$ consisting of eigenvectors of $W$ such that $W e_{k}=\lambda_{k} e_{k}, \lambda_{k}>0$. Let $P_{n}$ be the orthogonal projection onto the subspace generated by $\left\{e_{1}, \cdots, e_{n}\right\}$.

Let $\nu=\overline{W(\gamma)}$ and let $\epsilon>0$. Then for $m$ and $n$ large enough

$$
\begin{aligned}
& \epsilon>\gamma\left\{x:\left\|P_{n} x-P_{m} x\right\|>\epsilon\right\} \\
& =\nu\left\{x:\left\|A P_{n} x-A P_{m} x\right\|_{B}>\epsilon\right\} .
\end{aligned}
$$

Therefore $A P_{n} x$ converges in $\nu$-measure. Since $A$ is closed and $P_{n} x \rightarrow x$, it follows that $A P_{n} x \rightarrow A x$ in $\nu$-measure. But $\nu\{x \in$ $\left.H:\left\|A P_{n} x-A x\right\|_{B}>\epsilon\right\}=\mu\left\{x \in B:\left\|A P_{n} T x-x\right\|_{B}>\epsilon\right\}$ so $A P_{n} T x \rightarrow x$ in $\mu$-measure on $B$.

Now,

$$
\begin{aligned}
A P_{n} T x & =A \sum_{k=1}^{n}\left\langle T x, e_{k}\right\rangle e_{k} \\
& =\sum_{k=1}^{n}\left\langle x, T^{*} e_{k}\right\rangle A e_{k} \\
& =\sum_{k=1}^{n}\left\langle x, \lambda_{k} f_{k}\right\rangle A e_{k} \\
& =\sum_{k=1}^{n}\left\langle x, f_{k}\right\rangle S e_{k} .
\end{aligned}
$$


If $\gamma$ is the standard Gaussian cylinder measure on $H$ we obtain the following well known $([5,7])$

COROLlary 3.4. With the same hypotheses as Theorem 3.3 and $\gamma$ the standard Gaussian cylinder measure on $\mathrm{H}$

$$
\sum_{k=1}^{n}\left\langle x, f_{k}\right\rangle S e_{k} \rightarrow x \quad \mu \text {-almost everywhere. }
$$

Proof. The random variables $\left\langle\cdot, f_{k}\right\rangle$ on $(B, \mu)$ are independent (see [5]) so by the well known theorem of P. Levy (see [1] Theorem 5.3.4, p. 120 and its proof), convergence in measure implies almost everywhere convergence.

\section{REFERENCES}

1. K. L. Chung, A course in probability theory, Second Edition, Academic Press, New York, 1974.

2. L. Gross, Measurable functions on Hilbert space, Trans. Amer. Math. Soc., 105 (1962), 372-390.

3. _- Abstract Wiener spaces, Proc. 5th Berkeley Symp. on Math. Stat. and Prob., University of California press, 1965.

4. J. Kuelbs, Gaussian measures on a Banach space, J. Functional Analysis, 5 (1970), 354-367.

5 . - Expansions of vectors in a Banach space related to Gaussian measures, Proc. Amer. Math Soc., 27, No. 2 (1971), 364-370.

6. H. H. Kuo, Gaussian measures in Banach spaces, Lecture Notes in Math. No. 463, SpringerVerlag, Berlin, 1975.

7. B. S. Rajput, On Gaussian measures in certain locally convex spaces, J. Multivariate Anal., 2 (1972), 282-306.

8. L. Schwartz, Measure cylindriques et applications radonifiantes, J. Fac. Sci., Univ. Tokyo, 18 No. 12 (1971), 139-286.

9. - Radon measures on arbitrary topological spaces and cylindrical measures, Tata Institute of Fundamental Research, Oxford Univerity Press, 1973.

10. A. Versik, Duality in the theory of measure in linear spaces, Soviet Math. Dokl. 7 (1966), $1210-1214$.

Received November 23, 1976.

Hunter College, CUNy

NEW YORK, NY 10021. 



\section{Pacific Journal of Mathematics \\ Vol. 70, No. $1 \quad$ September, 1977}

William H. Barker, Noether's theorem for plane domains with hyperelliptic

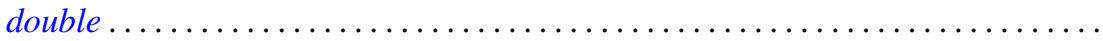

Michael James Beeson, Non-continuous dependence of surfaces of least area on the boundary curve ...................................... 11

Horst Behncke, Functions acting in weighted Orlicz algebras . . . . . . . . . . . . 19

Howard Edwin Bell, A commutativity study for periodic rings . . . . . . . . . . . 29

Peter Botta and Stephen J. Pierce, The preservers of any orthogonal group ....... 37

Douglas S. Bridges, The constructive Radon-Nikodým theorem ............. 51

James Dennis Brom, The theory of almost periodic functions in constructive

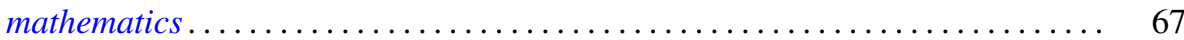

N. Burgoyne and C. Williamson, Semi-simple classes in Chevalley type groups ....

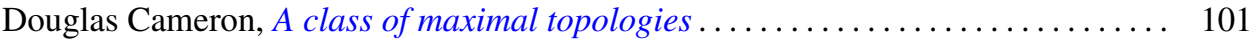

L. Carlitz, Enumeration of doubly up-down permutations . . . . . . . . . . . . . . 105

Paul Robert Chernoff, The quantum n-body problem and a theorem of

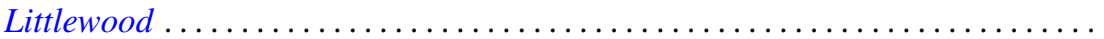

Jo-Ann Deborah Cohen, Locally bounded topologies on $F(X) \ldots \ldots \ldots \ldots \ldots \ldots$

Heinz Otto Cordes and Robert Colman McOwen, Remarks on singular elliptic

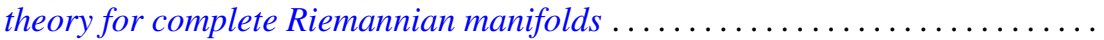

Micheal Neal Dyer, Correction to: "Rational homology and Whitehead

products"

Robert Fernholz, Factorization of Radonifying transformations

Lawrence Arthur Fialkow, A note on quasisimilarity. II ...... . .

Harvey Charles Greenwald, Lipschitz spaces of distributions on the surface of unit

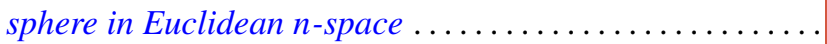

Albrecht Irle, On the measurability of conditional expectations

Tom (Roy Thomas Jr.) Jacob, Matrix transformations involving simple sequence

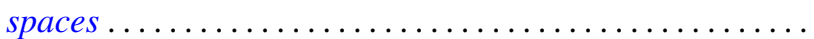

A. Katsaras, Continuous linear maps positive on increasing continuous

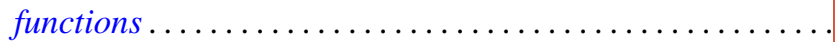

Kenneth Kunen and Judith Roitman, Attaining the spread at cardinals of cofinality

Lawrence Louis Larmore and Robert David Rigdon, Enumerating normal bundles

of immersions and embeddings of projective spaces ...... . .

Ch. G. Philos and V. A. Staïkos, Asymptotic properties of nonoscillatory solutions of differential equations with deviating argument .

Peter Michael Rosenthal and Ahmed Ramzy Sourour, On operator algebras containing cyclic Boolean algebras...

Polychronis Strantzalos, Strikt fast gleichgradig-stetige und eigentliche

Aktionen ...

Glenn Francis Webb, Exponential representation of solutions to an abstract

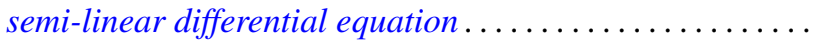

